\title{
MiR-378 Has the Capacity to Serve as a Diagnostic Biomarker for Prostate Cancer Patient
}

\author{
Shuangqing Cao ( $\nabla$ bhvgfw@163.com ) \\ Harrison International Peace Hospital https://orcid.org/0000-0002-4017-6014 \\ Lei Zheng \\ Harrison International Peace Hospital
}

Research article

Keywords: MiR-378, Prostate cancer, Diagnosis, ROC

Posted Date: August 11th, 2020

DOI: https://doi.org/10.21203/rs.3.rs-53222/v1

License: (9) This work is licensed under a Creative Commons Attribution 4.0 International License. Read Full License 


\section{Abstract}

Background: This study aimed to examine the expression of serum miR-378 in prostate cancer (PCa) patients and healthy individuals and to identify the value of miR-378 in PCa diagnosis.

Methods: The expression of serum miR-378 between groups was compared by t-test. The association between miR-378 expression and clinical characteristics of PCa patients was assessed using Chi-square test. The diagnostic value of serum miR-378 in PCa was estimated by the receiver operating characteristic (ROC) analysis.

Results: The expression level of serum miR-378 in PCa patients was significantly lower than that in healthy individuals $(P<0.0001)$. MiR-378 expression was affected by positive AR $(P=0.004)$, large Gleason score $(P=0.013)$ and advanced TNM stage $(P=0.020)$, however, it had no relationship with age, serum PSA, NED rate and urine retention (all, $P>0.05$ ). The ROC curve showed that the optimal cutoff value was 1.845 , giving the sensitivity and specificity of $75.21 \%$ and $89.77 \%$, respectively. Besides, the area under the ROC curve (AUC) was 0.894 , indicating serum miR-378 was of great diagnostic value in screening $\mathrm{PCa}$ patients from healthy controls $(P<0.0001,95 \% \mathrm{Cl}=0.852-0.936)$.

Conclusions: Taken together, the increased expression of serum miR-378 might act as a potential biomarker for PCa diagnosis.

\section{Background}

Prostate cancer $(\mathrm{PCa})$ is one of the most frequently and commonly encountered malignancies among males, especially in the European and American regions [1, 2]. In addition, the incidence rate of PCa in Asian regions has significantly increased in recent years though advanced therapies including surgery, hormone therapy, chemotherapy and radiation therapy had been applied in PCa patients $[3,4]$. Besides, it has been reported that PCa is one of the leading causes for cancer-related deaths in men $[5,6]$. According to the guidelines of European Association of Urology and American Urological Association, the current diagnosis of PCa mainly depends on digital rectal examination (DRE) and increased prostate specific antigen (PSA), which have several limitations, such as low diagnostic specificity and missed diagnosis [7, 8]. What's more, the development of PCa was a slow process and majority of PCa patients are present with no obvious symptoms at early stage $[5,9]$. Therefore, it is in urgent need to find novel biomarkers with high sensitivity and specificity to diagnosed PCa at early stage.

MicroRNAs (miRNAs) are a class of short, endogenous, single-stranded, conserved non-coding RNAs that are about 17-25 nucleotides in length in eucaryote cells [10,11]. They can bind to the 3 -untranslated regions ( $3^{\prime}-U T R$ ) of a variety of genes to regulate their expression through sequence-specific base paring at post-transcription level $[12,13]$. A large number of studies have demonstrate that miRNAs play crucial roles in biological processes, such as cell proliferation, migration, invasion, apoptosis and oncogenesis [14-16]. MiR-378, as a member of miRNA family, has also been reported to be involved in cell survival, angiogenesis, and tumor growth $[17,18]$. Besides, a growing number of investigations have claimed that 
abnormal expression of miR-378 are related with the occurrence of different cancers, such as ovarian cancer [19]. Considerable studies have demonstrated the candidate role of miRNAs in tumor diagnosis and prognosis. However, until now, the diagnostic role of miR-378 in PCa patients was still unclear.

In the present study, we aimed to determine the expression of serum miR-378 in PCa patients and further to assess its diagnostic value for this disease.

\section{Methods}

Patients and specimens

A total of 117 patients who were pathologically diagnosed as PCa were recruited from Harrison International Peace Hospital. Patients accompanied with other prostatic diseases, such as prostatitis, were excluded from the study. The collected patients received no chemo- or radio- therapy before this investigation. In addition, 88 blood donors in the same hospital were enrolled as healthy controls. An aliquot of $5 \mathrm{ml}$ peripheral blood was collected from all the participants to prepare serum samples. The serum samples were stored in EDTA-containing tubes for later use. This study was conducted with the approval of the Ethics Committee of Harrison International Peace Hospital. The informed consent had obtained from each participant in advance.

Quantitative real-time polymerase chain reaction (qRT-PCR)

Total RNA was extracted from serum samples by Qiagen miRNeasy Mini Kit (Qiagen, GmbH, Germany) following the manufacture's instruction. Then the total RNA was used to synthesize cDNA according the the TaqMan MicroRNA Assay protocol (Applied Biosystems). Finally, the real-time PCR amplification was performed using the Applied Biosystems 7599 instrument under optimal conditions. The expression of serum miR-378 was calculated using the $2^{-\Delta \Delta C t}$ method. In addition, $U 6$ was adopted as an internal reference.

\section{Statistical analysis}

All data in the study were statistically analyzed using Sigmaplot 12.5 and GraphPad Prism 5.0 software. The student's t-test was used to compare the expression difference of miR-378 between groups. Chisquare test was used to describe the relationship of miR-378 expression and clinical parameters. The receiver operating characteristics (ROC) curve was plotted to calculate the area under the curve (AUC) as well as the sensitivity and specificity of miR-378 in PCa patients. $P$ value was considered to be significant if it was less than 0.05 .

\section{Results}

Down-regulated expression of serum miR-378 in PCa patients

The expression of miR-378 in PCa patients and healthy controls was determined using qRT-PCR. The relative expression level of serum miR-378 in PCa patients was $1.824 \pm 0.576$, while that in healthy 
individuals was $3.101 \pm 0.875$. As shown in Fig. 1, miR-378 was significantly decreased in PCa serum compared with healthy controls $(P<0.0001)$.

Relationship between miR-378 expression and clinical features of PCa patients

To explore the relationship of miR-378 expression with clinical characteristics of PCa patients, the $\chi^{2}$ test was conducted. Patients were classified into high expression group $(n=59)$ and low expression group $(n$ $=58$ ) according to the median expression level (1.75) of serum miR-378. As listed in Table 1, the increased expression of serum miR-378 was closely related with AR status $(P=0.004)$, Gleason score $(P$ $=0.013)$ and TNM stage $(P=0.020)$. However, no relationship was found between miR-378 expression and age $(P=0.117)$, serum PSA level $(P=0.298)$, urine retention $(P=0.117)$ and NET rate $(P=0.079)$. 
Table 1

Relationship of miR-378 expression and clinical characteristics of PCa patients

\begin{tabular}{|c|c|c|c|c|c|}
\hline \multirow[t]{2}{*}{ Clinical features } & \multirow{2}{*}{$\begin{array}{l}\text { Case } \\
\text { NO. }\end{array}$} & \multicolumn{2}{|c|}{ Expression } & \multirow{2}{*}{$x^{2}$} & \multirow[t]{2}{*}{$P$ value } \\
\hline & & High & Low & & \\
\hline Age & & & & 2.462 & 0.117 \\
\hline$\leqq 65$ & 61 & 35 & 26 & & \\
\hline$>65$ & 56 & 24 & 32 & & \\
\hline Serum PSA (ng/ml) & & & & 1.085 & 0.298 \\
\hline$\leqq 10$ & 67 & 31 & 36 & & \\
\hline$>10$ & 50 & 28 & 22 & & \\
\hline NED rate & & & & 3.095 & 0.079 \\
\hline$\leqq 40 \%$ & 51 & 21 & 30 & & \\
\hline$>40 \%$ & 66 & 38 & 28 & & \\
\hline Urine retention & & & & 2.463 & 0.117 \\
\hline Present & 63 & 36 & 27 & & \\
\hline Absent & 54 & 23 & 31 & & \\
\hline AR status & & & & 8.377 & 0.004 \\
\hline Positive & 65 & 25 & 40 & & \\
\hline Negative & 52 & 34 & 18 & & \\
\hline Gleason score & & & & 6.223 & 0.013 \\
\hline$\leqq 6$ & 57 & 22 & 35 & & \\
\hline$>7$ & 60 & 37 & 23 & & \\
\hline TNM stage & & & & 5.431 & 0.020 \\
\hline $\mathbb{Q}, \mathbb{Z}$ & 64 & 26 & 38 & & \\
\hline Q,, & 53 & 33 & 20 & & \\
\hline
\end{tabular}

Diagnostic performance of serum miR-378 in $\mathrm{PCa}$

The ROC analysis was conducted to identify the diagnostic role of serum miR-378 in PCa. As shown in Fig. 2, the optimal cutoff point was 1.845 , giving the sensitivity of $75.21 \%$ and the specificity of $89.77 \%$. 
Besides, the area under ROC curve (AUC) was 0.894 , suggesting serum miR-378 could discriminate PCa patients from healthy individuals $(P<0.0001)$.

\section{Discussion}

PCa is one of the most common male malignant tumors in the world. Due to the large population, aging, or changes of life style, the number of PCa patients has been increased greatly in recent years [20]. Patients at early stage are present with no obvious symptoms, which indicates that there might be local infiltration or distant metastasis when symptoms appear, such as urine retention, difficult urination and hematuria. In recent years, the detection rate of PCa has been significantly elevated due to the application of serum PSA and DRE in clinic. Nevertheless, there are several limitations in these typical screening methods. And it is known to all that tumors at early stages have more opportunities to be cured than the advanced ones. Therefore, more and novel specific biomarkers are needed to early diagnose PCa. In the study of Wu et al., urine PCA3 was used as a diagnostic biomarker for PCa [21]. Casanova-salas et al. demonstrated that miR-187 and miR-182 has great capacity for diagnosing PCa [22]. In this study, we were engaged in finding more novel biomarkers to better improve the PCa diagnosis.

MiRNAs has been reported to be implicated with plenty of physical and biological processes through regulating the expression of target genes. It is said that miRNAs are abundant in serum, plasma and other body fluids with high stability [23]. Besides, miRNAs have similar signatures between men and women with different ages. In the last decade, numerous miRNAs have been investigated as prognostic and diagnostic biomarkers and therapeutic targets. Cao et al. revealed that miR-454 overexpression was correlated with unfavorable prognosis for patients with triple-negative breast cancer [24]. In the study of Wei et al., they reported that miR-1 was significantly downregulated in recurrent PCa tissues and it can function as an independent predictive factor for PCa [25]. Moreover, Want et al. showed that miR-378 was related with poor prognosis as well as tumor invasiveness of patients with glioma [26]. In the present study, we attempted to detect the diagnostic role of serum miR-378 in PCa.

Previous studies have demonstrated that serum miR-378 was significantly upregulated in several cancers, including gastric cancer, renal cell carcinoma and PCa [23, 27, 28]. At present, we first compared the expression levels of serum miR-378 in PCa patients and healthy individuals, and our findings were highly in accordance with previous results, indicating miR-378 might be related with the progression of $\mathrm{PCa}$. The Chi-square test elucidated that serum miR-378 up-regulation was influenced by AR status, Gleason score and TNM stage. Based on the above findings, we further determined the diagnostic role of serum miR-378 in PCa using ROC analysis and the results showed a high AUC value for serum miR-378 in PCa diagnosis, indicating serum miR-378 could distinguish PCa patients from healthy controls.

In this study, we identified the diagnostic role of serum miR-378 in PCa for the first time. However, the precise mechanisms of serum miR-378 on PCa pathogenesis are still not clear. In the study of Qi et al., they found miR-378 was down-regualted in PCa tissues and validated MAPK1 was a direct target of miR378 in human PCa [29]. Besides, Avgeris et al. also observed the reduction of miR-378 was predicted to 
target both KLK2 and KLK4 and increases the risk of PCa progression [30]. Therefore, more and further investigations are required for future studies.

\section{Conclusions}

In conclusion, we confirmed the down-regulation of serum miR-378 in PCa patients compared with healthy individuals. Positive AR, large Gleason score and advanced TNM stage were associated with miR378 expression. Furthermore, our results identified the potential of serum miR-378 as a promising diagnostic biomarker for PCa.

\section{Abbreviations}

prostate cancer $(\mathrm{PCa})$

receiver operating characteristic (ROC)

area under the ROC curve (AUC)

digital rectal examination (DRE)

prostate specific antigen (PSA)

MicroRNAs (miRNAs)

3'-untranslated regions (3'-UTR)

Quantitative real-time polymerase chain reaction (qRT-PCR)

\section{Declarations}

\section{Ethics approval and consent to participate}

This study was supported by the Ethics Committee of Harrison International Peace Hospital and also has been carried out in accordance with the World Medical Association Declaration of Helsinki.

The subjects had been informed the objective. Certainly, written consents were signed by every subject in this study.

\section{Consent for publication}

We obtaining permission from participants to publish their data.

\section{Availability of data and materials}


The datasets used and/or analysed during the current study are available from the corresponding author on reasonable request.

\section{Competing interests}

The authors declare that they have no competing interests.

Funding Not applicable.

\section{Authors' contributions}

S.C., L.Z. design of the work; S.C., L.Z. the acquisition, analysis, S.C., L.Z. interpretation of data; S.C., L.Z. the creation of new software used in the work; S.C., L.Z. have drafted the work or substantively revised it. All authors read and approved the final manuscript.

Acknowledgements Not applicable.

\section{References}

1. Cao Y, Nimptsch K, Shui IM, Platz EA, Wu K, Pollak MN, Kenfield SA, Stampfer MJ, Giovannucci EL. Prediagnostic plasma IGFBP-1, IGF-1 and risk of prostate cancer. International journal of cancer Journal international du cancer. 2015;136(10):2418-26.

2. Huang L, Li M, Wang D, He J, Wu W, Zeng Q, Li J, Xiao M, Hu J, He Y, et al. Overexpressed Rce1 is positively correlated with tumor progression and predicts poor prognosis in prostate cancer. Human pathology. 2016;47(1):109-14.

3. Tahara H, Naito H, Kise K, Wakabayashi T, Kamoi K, Okihara K, Yanagisawa A, Nakai Y, Nonomura N, Morii E, et al. Evaluation of PSF1 as a prognostic biomarker for prostate cancer. Prostate Cancer Prostatic Dis. 2015;18(1):56-62.

4. Jiang CY, Gao Y, Wang XJ, Ruan Y, Bei XY, Wang XH, Jing YF, Zhao W, Jiang Q, Li J, et al. Long noncoding RNA Inc-MX1-1 is associated with poor clinical features and promotes cellular proliferation and invasiveness in prostate cancer. Biochem Biophys Res Commun. 2016;470(3):721-7.

5. Kong HY, Byun J. Screening and characterization of a novel RNA aptamer that specifically binds to human prostatic acid phosphatase and human prostate cancer cells. Mol Cells. 2015;38(2):171-9.

6. Stallone G, Cormio L, Netti GS, Infante B, Selvaggio O, Fino GD, Ranieri E, Bruno F, Prattichizzo C, Sanguedolce $F$, et al. Pentraxin 3: a novel biomarker for predicting progression from prostatic inflammation to prostate cancer. Cancer research. 2014;74(16):4230-8.

7. Heidegger I, Hofer J, Luger M, Pichler R, Klocker H, Horninger W, Steiner E, Jochberger S, Culig Z. Is Eotaxin-1 a serum and urinary biomarker for prostate cancer detection and recurrence? Prostate. 2015;75(16):1904-9.

8. Lucarelli G, Rutigliano M, Bettocchi C, Palazzo S, Vavallo A, Galleggiante V, Trabucco S, Di Clemente D, Selvaggi FP, Battaglia M, et al. Spondin-2, a secreted extracellular matrix protein, is a novel 
diagnostic biomarker for prostate cancer. The Journal of urology. 2013;190(6):2271-7.

9. Reiter RJ, Tan DX, Manchester LC, Korkmaz A, Fuentes-Broto L, Hardman WE, Rosales-Corral SA, Qi $W$. A walnut-enriched diet reduces the growth of $L N C a P$ human prostate cancer xenografts in nude mice. Cancer investigation. 2013;31(6):365-73.

10. Qian J, Lin J, Qian W, Ma JC, Qian SX, Li Y, Yang J, Li JY, Wang CZ, Chai HY, et al. Overexpression of miR-378 is frequent and may affect treatment outcomes in patients with acute myeloid leukemia. Leukemia research. 2013;37(7):765-8.

11. Bi L, Yang Q, Yuan J, Miao Q, Duan L, Li F, Wang S. MicroRNA-127-3p acts as a tumor suppressor in epithelial ovarian cancer by regulating the BAG5 gene. Oncol Rep. 2016;36(5):2563-70.

12. $X u L$, Shen $B$, Chen $T$, Dong $P$ : miR-203 is involved in the laryngeal carcinoma pathogenesis via targeting VEGFA and Cox-2. OncoTargets and therapy 2016, 9:4629-4637.

13. Ma J, Lin J, Qian J, Qian W, Yin J, Yang B, Tang Q, Chen X, Wen X, Guo H, et al. MiR-378 promotes the migration of liver cancer cells by down-regulating Fus expression. Cellular physiology biochemistry: international journal of experimental cellular physiology biochemistry pharmacology. 2014;34(6):2266-74.

14. Xiao J, Lin HY, Zhu YY, Zhu YP, Chen LW. MiR-126 regulates proliferation and invasion in the bladder cancer BLS cell line by targeting the PIK3R2-mediated PI3K/Akt signaling pathway. OncoTargets therapy. 2016;9:5181-93.

15. Sun Y, Zhao J, Yin X, Yuan X, Guo J, Bi J. miR-297 acts as an oncogene by targeting GPC5 in lung adenocarcinoma. Cell proliferation. 2016;49(5):636-43.

16. Xie N, Wang C, Zhuang Z, Hou J, Liu X, Wu Y, Liu H, Huang H. Decreased miR-320a promotes invasion and metastasis of tumor budding cells in tongue squamous cell carcinoma. Oncotarget. 2016;7(40):65744-57.

17. Wang KY, Ma J, Zhang FX, Yu MJ, Xue JS, Zhao JS. MicroRNA-378 inhibits cell growth and enhances L-OHP-induced apoptosis in human colorectal cancer. IUBMB Life. 2014;66(9):645-54.

18. Xing Y, Hou J, Guo T, Zheng S, Zhou C, Huang H, Chen Y, Sun K, Zhong T, Wang J, et al. microRNA378 promotes mesenchymal stem cell survival and vascularization under hypoxic-ischemic conditions in vitro. Stem Cell Res Ther. 2014;5(6):130.

19. Chan JK, Kiet TK, Blansit K, Ramasubbaiah R, Hilton JF, Kapp DS, Matei D. MiR-378 as a biomarker for response to anti-angiogenic treatment in ovarian cancer. Gynecol Oncol. 2014;133(3):568-74.

20. Chen W, Zheng R, Zhang S, Zhao P, Li G, Wu L, He J. Report of incidence and mortality in China cancer registries, 2009. Chinese journal of cancer research $=$ Chung-kuo yen cheng yen chiu. 2013;25(1):10-21.

21. Xue WJ, Ying XL, Jiang JH, Xu YH: Prostate cancer antigen 3 as a biomarker in the urine for prostate cancer diagnosis: a meta-analysis. Journal of cancer research and therapeutics 2014, 10 Suppl:C218-221.

22. Casanova-Salas I, Rubio-Briones J, Calatrava A, Mancarella C, Masia E, Casanova J, Fernandez-Serra A, Rubio L, Ramirez-Backhaus M, Arminan A, et al. Identification of miR-187 and miR-182 as 
biomarkers of early diagnosis and prognosis in patients with prostate cancer treated with radical prostatectomy. The Journal of urology. 2014;192(1):252-9.

23. Redova M, Poprach A, Nekvindova J, Iliev R, Radova L, Lakomy R, Svoboda M, Vyzula R, Slaby O. Circulating miR-378 and miR-451 in serum are potential biomarkers for renal cell carcinoma. Journal of translational medicine. 2012;10:55.

24. Cao ZG, Li JJ, Yao L, Huang YN, Liu YR, Hu X, Song CG, Shao ZM. High expression of microRNA-454 is associated with poor prognosis in triple-negative breast cancer. Oncotarget. 2016;7(40):64900-9.

25. Wei W, Leng J, Shao H, Wang W. MiR-1, a Potential Predictive Biomarker for Recurrence in Prostate Cancer After Radical Prostatectomy. Am J Med Sci. 2017;353(4):315-9.

26. Li B, Wang Y, Li S, He H, Sun F, Wang C, Lu Y, Wang X, Tao B. Decreased expression of miR-378 correlates with tumor invasiveness and poor prognosis of patients with glioma. Int J Clin Exp Pathol. 2015;8(6):7016-21.

27. Liu H, Zhu L, Liu B, Yang L, Meng X, Zhang W, Ma Y, Xiao H. Genome-wide microRNA profiles identify miR-378 as a serum biomarker for early detection of gastric cancer. Cancer letters. 2012;316(2):196203.

28. Nguyen HC, Xie W, Yang M, Hsieh CL, Drouin S, Lee GS, Kantoff PW. Expression differences of circulating microRNAs in metastatic castration resistant prostate cancer and low-risk, localized prostate cancer. Prostate. 2013;73(4):346-54.

29. Chen QG, Zhou W, Han T, Du SQ, Li ZH, Zhang Z, Shan GY, Kong CZ. MiR-378 suppresses prostate cancer cell growth through downregulation of MAPK1 in vitro and in vivo. Tumour biology: the journal of the International Society for Oncodevelopmental Biology Medicine. 2016;37(2):2095-103.

30. Avgeris M, Stravodimos K, Scorilas A. Loss of miR-378 in prostate cancer, a common regulator of KLK2 and KLK4, correlates with aggressive disease phenotype and predicts the short-term relapse of the patients. Biological chemistry. 2014;395(9):1095-104.

\section{Figures}




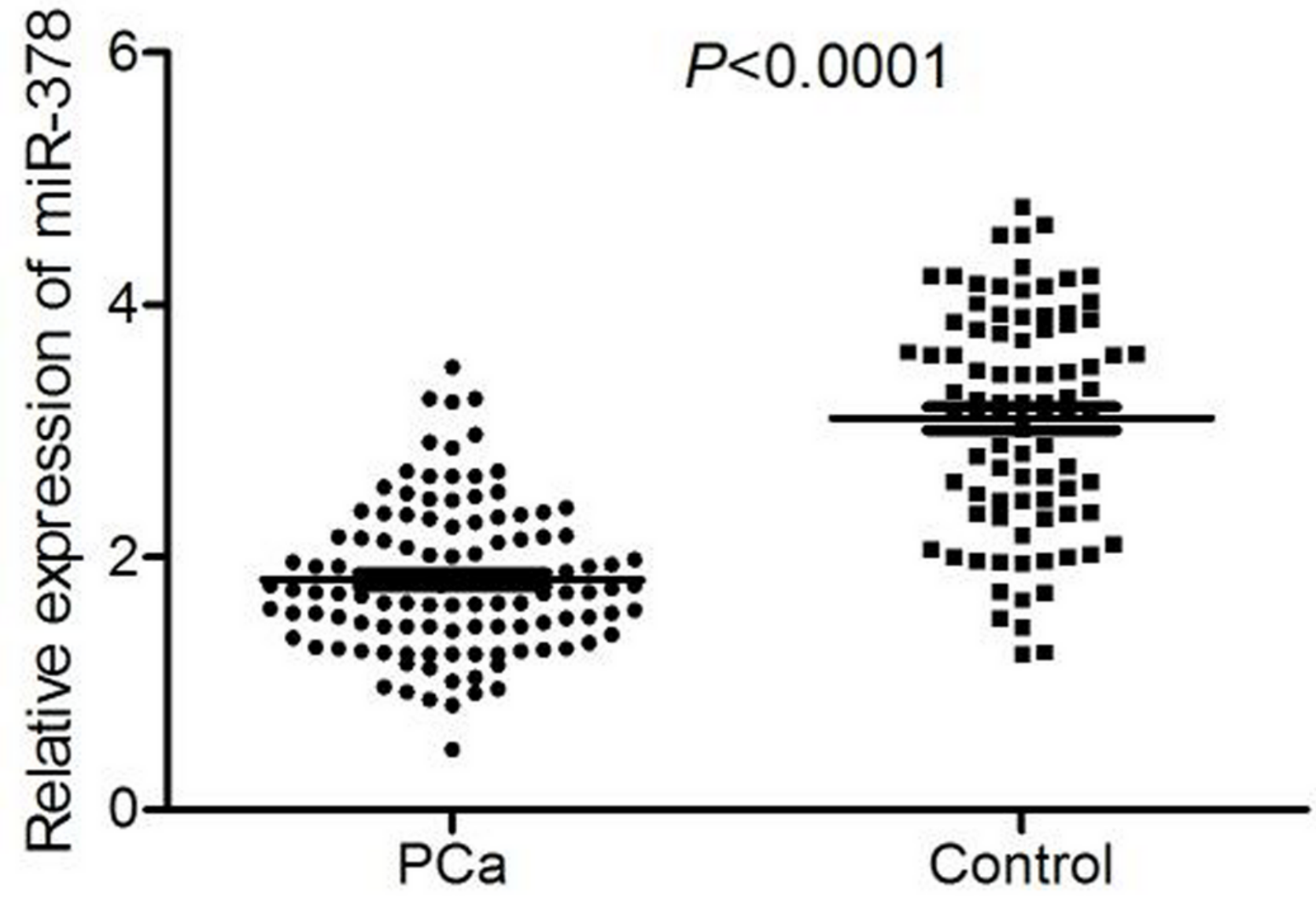

Figure 1

Expression of serum miR-378 in PCa patients and healthy controls. The result showed that serum miR378 level was significantly lower in $\mathrm{PCa}$ patients than that in healthy controls $(\mathrm{P}<0.0001)$. 


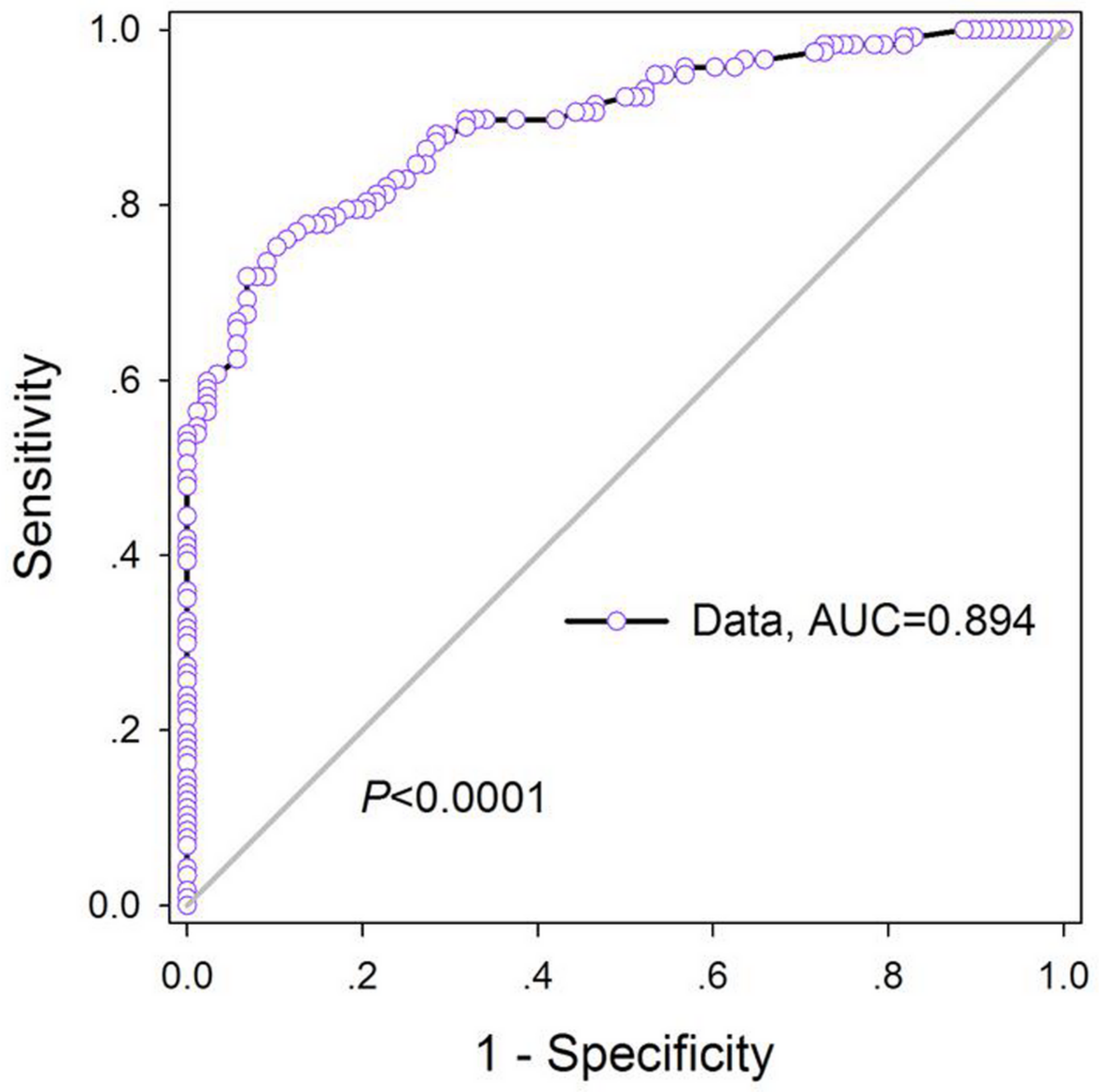

Figure 2

ROC curve for PCa patients with different serum miR-378 expression. It could be concluded that serum miR-378 could act as a diagnostic biomarker in PCa with the AUC of $0.894(P<0.0001)$. 